\title{
Traduire
}

Une autre perspective sur r tr traduction

Revue française de la traduction

$243 \mid 2020$

Les arts du spectacle

\section{Quand la traduction entre en jeu}

\section{Laurence Van Goethem}

\section{(2) OpenEdition}

\section{Journals}

Édition électronique

URL : http://journals.openedition.org/traduire/2137

DOI : 10.4000/traduire.2137

ISSN : 2272-9992

Éditeur

Société française des traducteurs

Édition imprimée

Date de publication : 15 décembre 2020

Pagination : 44-52

ISSN : 0395-773X

\section{Référence électronique}

Laurence Van Goethem, «Quand la traduction entre en jeu », Traduire [En ligne], 243 | 2020, mis en ligne le 15 décembre 2020, consulté le 31 décembre 2020. URL : http://journals.openedition.org/ traduire/2137; DOI : https://doi.org/10.4000/traduire.2137 


\section{Quand la traduction entre en jeu}

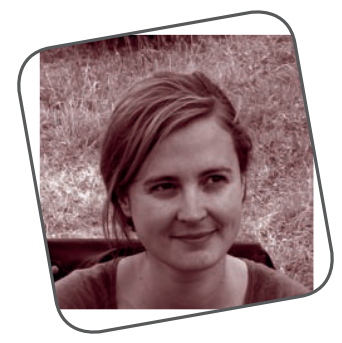

\section{Laurence Van Goethem}

Longtemps la traduction théâtrale a été assimilée à la traduction littéraire: c'est le règne de la primauté du texte écrit sur la performance, inscrite dans une temporalité déterminée, par définition éphémère. Le texte théâtral est par conséquent comparable au texte littéraire, et la traduction théâtrale considérée comme «semblable» à la traduction littéraire: "La nature de l'acte de traduire est à mon avis toujours la même», disait Antoine Vitez.

Bien entendu, le traducteur de théâtre se doit d'être attentif au rythme, à la diction, au registre; au mieux, il prendra en compte la mise en scène à venir et participera, si possible, aux répétitions où il est encore susceptible de modifier son texte. Dans cette perspective, peu de différences existent avec la traduction d'un roman ou d'un poème, hormis le rapport dialectique qui peut s'instaurer entre l'écriture et la mise en scène (voir le principe de «jouabilité» souvent débattu). Le traducteur est dans ce cas un linguiste qui se situe dans le domaine littéraire et qui fait une incursion dans l'univers théâtral.

Or le théâtre n'est pas à proprement parler de la littérature. Il est un art qui allie «texte» et «corps»; il est un «verbocorps», et il n'a de sens que s'il est exposé face à un public. Trois éléments qui participent de l'éclosion de cet art qualifié de «vivant». 


\section{Les variations infinies}

Avant toute entreprise de traduction et dans les mêmes termes pour toute œuvre, littéraire ou non, se posent les questions du "pourquoi traduire», du «que traduire» et de «qui traduit ${ }^{1} »$. Les réponses à la première question sont multiples: pour franchir des frontières, frotter une culture étrangère à la sienne, dialoguer entre humains, mieux se comprendre: «traverser ce qui est différent, l'interroger et le connaître» (Antonio Prete ${ }^{2}$ ), ou tout simplement «faire connaître ce que j'aime» (Antoine Vitez ${ }^{3}$ ).

La question de «qui traduit» au théâtre est intrinsèquement liée aux autres (que traduit-on, et pourquoi?), à quoi nous pourrions ajouter «pour qui? », car on traduit au théatre aussi en fonction du public, et il peut arriver que les acteurs modifient légèrement le texte pour mieux coller à l'actualité ou au lieu de la représentation. Ainsi la traduction est ouverte, souple, susceptible d'être retouchée en fonction des circonstances.

Dans de nombreuses pièces contemporaines, elle est même réalisée oralement sur scène, totalement intégrée à la mise en scène, et le traducteur assimilé à un acteur (ou vice versa).

\section{Jérôme Bel, traduction en transition}

Dans Disabled de Jérôme $\mathrm{Bel}^{4}$, un spectacle interprété par une troupe professionnelle, porteuse de divers handicaps mentaux, une actrice traduit sur scène le propos des comédien.nes (qui jovent leur propre rôle). Cette actricetraductrice est (apparemment) la seule "non invalide». Elle est là pour lier les scènes entre elles, expliquer la mise en œuvre de la production, donner le nom du metteur en

1. Voir Margaret Tomarchio, "Le théâtre en traduction: quelques réflexions sur le rôle du traducteur (Beckett, Pinter) », in Palimpsestes, 3/1990.

2. Antonio Prete, Á l'ombre de l'autre langue, traduit de l'italien par Danièle Robert, Les éditions chemin de ronde, 2013, p. 11.

3. Antoine Vitez, Le Devoir de traduire, Entretien avec Georges Banu et Alain Girault, Actes Sud-Papiers, p. 22.

4. Disabled de Jérôme Bel, cour d'honneur du Festival d'Avignon, 2012. Le spectacle est en accès libre sur https://www.youtube.com/watch?v=1mloo wjpH2c\&t=4133s 
scène, s'adressant aussi bien au public (en anglais) qu'aux acteurs (en suisse allemand). La traduction, qui s'apparente ici à de l'interprétation, permet d'ouvrir la dramaturgie à diverses significations. Elle renforce aussi la dimension métathéâtrale de la pièce, l'aspect «fabrication» du théâtre en direct.

L'actrice-traductrice se présente d'emblée en son nom propre, comme une personne réelle: "Bonjour, je m'appelle Simone Truong, on m'a engagée comme assistante et traductrice parce que les acteurs ne parlent que suisse allemand, et Jérôme Bel pas.» Avec une voix très douce et une attitude calme, elle demeure immobile et impassible derrière un ordinateur, à une petite distance des autres comédien.nes. De ce fait, elle peut s'apparenter à une sorte d'automate et poser au passage la question des limites de la technologie dans la traduction et les rapports humains.

La fonction qu'elle remplit pendant la représentation, celle de prendre en charge l'interface entre les acteurs et le public, l'ancre dans le monde réel et scinde les deux univers, le «vrai» (le réel) et le "faux» (le fictif). Cependant, cette présence, qui peut sembler rassurante au début, estompe au fil du temps cette distinction, et l'on finit par inverser les notions de réel et d'irréel, et par poser en filigranes la question de la norme et de la normalité.

Son rôle permet également d'ouvrir une perspective de fond qui va au-delà des problèmes linguistiques et de la compréhension immédiate de la pièce: ces acteurs pourraient-ils être seuls sur scène? Ont-ils nécessairement besoin d'être accompagnés? Quel est leur degré d'autonomie? Les mêmes questions pourraient se poser pour les spectateurs, qui ont sans doute peu l'habitude de se retrouver face à un tel groupe de personnes atteintes de handicaps.

Ainsi, cette présence incarnée de la traductrice qui "fait corps» avec les comédien.nes plonge le public dans une légère inquiétude. Doublement éloigné de sa zone de confort par la langue étrangère (le suisse allemand) et par l'étrangeté des protagonistes, le spectateur est cependant rapidement entraîné par l'énergie déployée sur scène et oublie bien volontiers de distinguer la réalité de la fiction. 


\section{Ascanio Celestini, le dédoublement}

Le cas d'Ascanio Celestini ${ }^{5}$ nous semble assez original et exemplaire du «dédoublement» au théâtre.

Anthropologue de formation, A. Celestini poursuit avec une grande maîtrise la tradition du théâtre-récit en Italie. Ses spectacles se conçoivent à partir d'une minutieuse collecte de témoignages oraux qu'il restitue seul sur scène, ou accompagné par l'accordéoniste Gianluca Casadei. II porte la voix des marginaux, des exclus, des "derniers» qui, souvent caricaturés et «déshumanisés» dans la vie réelle, forment chez lui une communauté imaginaire attendrissante.

Lorsqu'il vient présenter ses spectacles en Belgique, il est accompagné sur scène par le comédien Patrick Bebi, qui interrompt fréquemment son récit pour le dire en français. P. Bebi incarne littéralement A. Celestini, imitant ses gestes, son intonation, le rythme de sa prosodie. Il jove en équilibre entre surtraductions et sous-traductions, intercalant à l'occasion et de façon improvisée un dialogue avec l'Italien. Le spectateur se retrouve face à un dédoublement de l'acteur/ personnage qui décale le sens du propos général de la pièce. Le résultat est comme doublement vivant, doublement authentique, alors que, dans l'ensemble, l'articulation des deux comédiens a quelque chose d'invraisemblable.

De son côté, David Murgia a découvert le théâtre de Celestini au Festival de Liège, alors qu'il était encore étudiant au conservatoire de cette ville (aujourd'hui École supérieure d'acteurs, Esact). Déjà marqué par le théâtre de Dario Fo à l'adolescence (le seul théâtre qu'il ait côtoyé à ce moment-là, dit-il), il trouve chez Celestini un maître à penser qui dépeint parfaitement sur scène les mécanismes sociaux pervers à l'œuvre dans nos sociétés, ceux qui, tout en muselant nos révoltes, exacerbent sans vergogne la lutte des classes. Les trois comédiens entretiennent depuis une dizaine d'années une amitié féconde, qui a mené à la recréation en français de certains spectacles de Celestini: Discours à la Nation, Laika

5. Voir Laurence Van Goethem, "Ascanio Celestini, subjectif, indirect, libre», in Alternatives théâtrales, $n^{\circ} 120,2014$. 
et Pueblo $0^{6}$. Dans ces reprises, le matériau dramaturgique de départ est traduit par Bebi, insufflé dans la voix et les gestes de Murgia, le tout sous la direction artistique de Celestini. L'enjeu étant de transposer à trois un univers singulier, tout en le réinventant.

Une des principales difficultés pour Murgia est de s'approprier ces récits et de les raconter comme s'ils venaient de lui. II s'agit moins de traduire des textes que toute une gestuelle, un rythme, une "géographie mentale personnelle ${ }^{7}$ » dans un rapport direct avec le public. Murgia commence toujours par écouter et observer attentivement les productions de l'auteur italien.

Une correspondance entre le spectacle original et sa reprise en français est ainsi instaurée dans la forme (des petites histoires qui s'imbriquent dans la grande Histoire), dans le rythme (très rapide, saccadé, les récits étant ponctués de "phrases-refrains») et dans le contenu - qu'on pourrait qualifier de politique, car fortement lié à "la cité». II est intéressant de noter les ajustements effectués sur le matériau original afin que la situation globale soit comprise par le public belge francophone.

En effet, David Murgia alterne une transposition très proche du texte et des prises de liberté totale. Prenons l'exemple du récit Les Pauvres, extrait de Pueblo, que le comédien a lu en français sur une chaîne de radio nationale belge, avant de l'intégrer au spectacle.

Fable typiquement celestinienne, Les Pauvres raconte avec ironie la façon dont les pauvres sont dépossédés de tout par les riches, même de leur propre révolte, et ne parviennent donc jamais à faire la révolution. Les références à l'actualité et la scansion du texte sont fondamentales pour bien "rendre» cette histoire.

II s'agit de privilégier la justesse de ton sur l'exactitude du sens. C'est là tout l'enjeu: garder le rythme, la sonorité et le sens global. Ainsi, des particularités typiquement italiennes sont parfois supprimées, comme l'évocation des braccianti di

6. Pueblo, de David Murgia et Ascanio Celestini, Théâtre national Wallonie-Bruxelles, du 5 au 9 janvier 2021.

7. Jean-Michel Desprats, Antoine Vitez, Le devoir de traduire, op. cit., p. 13. 
Di Vittorio nel foggiano (les ouvriers agricoles de Di Vittorio dans la province de Foggia ${ }^{8}$ ), et d'autres allusions sont ajoutées, qui font plus directement référence au territoire belge, comme dans cet extrait de la version française, absent de la version italienne:

Les questions les plus insensées des journalistes [...], qui osaient demander aux responsables syndicaux s'il était raisonnable et responsable de mener des grèves le jour où le viaduc de Bruxelles était fermé .

Ces adaptations libres ne peuvent se faire que dans un climat de confiance, fondé sur l'écoute et le dialogue, et un sentiment profond d'amitié. Ascanio et David créent, à partir d'un matériau de jeu source et après de longues heures de répétition, un langage commun. Chacun met à l'épreuve son identité, apportant sa langue, sa singularité d'acteur, d'auteur, de créateur, enrichissant l'œuvre dans une circulation fluide. La langue d'arrivée, bousculée par ce matériau source, est transformée et presque transcendée. Le théâtre retrouve ainsi son rôle de "laboratoire des gestes et des paroles de la société10 $»$.

\section{Teatro delle Albe: les classiques revisités}

Un autre genre de laboratoire de la langue est expérimenté depuis plus de trente ans par Marco Martinelli, lors d'ateliers pratiqués au sein de sa non scuola (non-école). L'auteur-metteur en scène italien, cofondateur du Teatro delle Albe (Ravenne), développe un travail de traduction-adaptation des classiques (Aristophane, Euripide, Sophocle, etc.) par des adolescents dans différents pays du monde (Italie, France, Belgique, États-Unis, Sénégal, etc.)"1. Sa pédagogie

8. Giuseppe di Vittorio (1892-1957), un fils d'ouvrier agricole qui deviendra un des pères du syndicalisme italien et un antifasciste célèbre.

9. L'italique est de notre fait. Cet extrait est tiré de la version radiophonique de ce récit lu par David Murgia sur La Première (RTBF), émission «Entrez sans frapper» du 10/10/2017.

10. Antoine Vitez, Le devoir de traduire, op. cit., p. 29.

11. Marco Martinelli, Aristophane dans les banlieves, traduit de l'italien par Laurence Van Goethem, Actes Sud-Papiers, 2020. L'auteur a réalisé un film sur son travail de recréation de la Divine Comédie par 150 enfants et adolescents de la banlieve de Nairobi (Kenya), The sky over Kibera, 2019. 
et les résultats qu'elle produit nous semblent une piste intéressante dans la "remise en vie» d'auteurs morts depuis longtemps, et dont les écrits pourraient paraître rébarbatifs. Prenons l'exemple d'Ubu roi, la célèbre pièce d'Alfred Jarry, adaptée en 2005 par Marco Martinelli, avec des adolescents issus d'une banlieve malfamée de Naples (Scampia), sous le titre Ubu sotto tiro. Après des semaines de répétitions, Simone, un garçon difficile (dont les parents étaient en prison), qui ne s'était encore jamais exprimé sauf dans des accès de colère, s'intéresse tout à coup à l'exercice en cours, qui consistait à trouver une façon de réaliser la scène où Père Ubu demande à ses «palotins» comment tuer le roi. Voici ce que raconte Marco Martinelli:

Je dis aux élèves: "La scène du texte n'est pas terrible, essayons de l'enrichir par notre imagination. Vous, comment vous le tueriez, le roi de Pologne? "Ils me donnèrent des solutions fantaisistes, mais rien de particulièrement ingénieux. À ce moment-là, Simone fut attentif et pour la première fois, il s'approcha de notre cercle. "Je sais, moi, comment faire crever le roi!» Je n'en crus pas mes oreilles: Simone avait parlé. "Très bien, Simone, et comment tu ferais? "I resta un moment en silence: "Je peux le dire qu'à toi, dans l'oreille.» L'occasion était trop belle, j'enfreignis les règles non écrites de la non-école, où les idées se communiquent collectivement et sont débattues tous ensemble, je laissai le groupe imaginer d'autres possibilités et je m'éloignai avec Simone dans un coin de l'Auditoire. II commença: "D'abord je lui arracherais les yeux et je les lui foutrais dans le nez.»Bon, et ensuite? "Ensuite, je lui arracherais les oreilles et je les flanquerais à la place de ses yeux.» D'accord... et puis? "Puis je lui perce les veines et je lui badigeonne la bite de rouge. Avec sa bite peinte en rouge, je lui fais un trou dans la tronche, dans le crâne, et je le lui fous dedans. Comme ça on démontre ce que le peuple a toujours dit.» C'est-à-dire, Simone? Qu'est-ce que le peuple a toujours dit? "Que le roi est une tête de noeud.»"

La joie et la créativité avec lesquelles les jeunes s'emparent de ces textes, les traduisant avec leurs propres mots pour les restituer "sauvagement» sur scène devant un public débordant d'enthousiasme sont particulièrement salutaires

12. Marco Martinelli, Aristophane dans les banlieves, op. cit., p. 107-108. 
et attestent de la vitalité que peut avoir le théâtre encore aujourd'hui.

\section{La traduction réconciliation}

Enfin, il nous semble important d'évoquer le travail réalisé par les troupes qui œuvrent dans des zones difficiles, parfois de conflits, comme le fait la compagnie Théâtre $\&$ Réconciliation dirigée par Frédérique Lecomte. Là, le matériau récolté pour les spectacles provient d'improvisations effectuées en amont. Un patient travail de traduction a lieu, aussi bien pendant les répétitions que sur scène lors des représentations publiques, car les participants choisis, parfois originaires d'ethnies en guerre, sont souvent non francophones et non scolarisés. Le théâtre joue alors un rôle "(ré)conciliateur» délicat: il est essentiel qu'une relation de confiance se nove entre la metteuse en scène et le traducteur, afin que circulent convenablement non seulement les informations, mais surtout le «texte intérieur» de chacun, les sous-entendus, les sentiments réfrénés. Pendant les représentations et les tournées dans différents pays africains, un acteur à tour de rôle se met légèrement à l'écart et traduit en direct ce que disent les autres, assumant le rôle ambigu de l'acteur jouant au traducteur, tout en l'étant réellement. On peut observer en détail cette traduction «incarnée» dans le court-métrage documentaire de Sanaz Azari ${ }^{13}$, qui est entièrement centré sur l'acteur-traducteur, laissant l'action de la pièce se dérouler hors champ.

Les traductions au théâtre renferment des richesses parfois insoupçonnées. Qu'elles soient effectuées in presentia, dans l'objectif d'offrir une compréhension immédiate, ou élaborées au cours de longues séances de répétitions, elles révèlent un dialogue visible ou sous-jacent qui stimule la performance. L'acteur-traducteur, dans ses différents degrés de visibilité (du traducteur absent à celui discrètement assis sur scène, jusqu'à celui qui endosse quasiment le rôle principal), met en lumière une dramaturgie de l'adaptation qui, plus que

13. Je suis le Pays, un film de Sanaz Azari, d'après les spectacles de Frédérique Lecomte, 2014, disponible en accès libre sur https://vimeo.com/155654469 
jamais, inscrit le théâtre dans un art «de l'autre $»^{14}$. L'objectif principal de la traduction n'est plus le texte dramatique en lui-même dans une sorte de sacralisation mortifère, mais la réalisation scénique à venir, dans une langue inventive, face à un public «vivant» et (ré)actif.

\section{I_van_goethem@yahoo.it}

Laurence Van Goethem a longtemps vécu en Italie où elle a enseigné le français avant de travailler dans le domaine de l'édition théâtrale à Bruxelles. En parallèle, elle se forme en traduction littéraire au Centre européen de traduction littéraire. En 2014, au théâtre Le Manège à Mons, elle rencontre Marco Martinelli et Ermanna Montanari, cofondateurs du Teatro delle Albe. Depuis lors, elle s'emploie à traduire en français les mots de Martinelli, œuvres de fiction (La Plage de Daura, éd. Emile Lansman, 2015) ou essais (Se Faire lieu, brèche dans le théâtre en 101 mouvements, éd. Alternatives théâtrales, 2018; Aristophane dans les banlieues. Pratiques de la non-école, Actes Sud-Papiers, 2020).

14. Voir Isabelle Pousseur, "Le théâtre, art de l'autre», in Alternatives théâtrales, hors-série $13,2013$. 